\title{
Desarrollo de un software web para la gestión de planes de negocios
}

\author{
Bernardo M. Choque-Tolmo', Marco A. Villalobos-Abarca ${ }^{2}$ y Raúl A. Herrera-Acuña ${ }^{2}$ \\ (1) CEO - Kuvemar Desarrollo Tecnológico, Membrillar 2623, Arica-Chile. (correo-e: bernardo.choque@gmail.com) \\ (2) Facultad de Ingeniería, Depto. de Ingeniería en Comp. e Inf., Universidad de Tarapacá, Casilla 7D, Arica-Chile. \\ (correo-e: mvillalo@uta.cl; rherrera@uta.cl)
}

Recibido Nov. 11, 2019; Aceptado Ene. 9, 2020; Versión final Feb. 28, 2020, Publicado Ago. 2020

\section{Resumen}

En este artículo se presenta el diseño y desarrollo de un sistema web para el apoyo en la generación de modelos y planes de negocios, orientado a emprendedores nuevos y establecidos, en el sector de micro y pequeñas empresas. El objetivo principal de este sistema es mejorar las posibilidades de acceso a financiamiento para los emprendedores, mediante una serie de herramientas de apoyo, como el uso de coaching experto y la administración de plantillas para postular a las principales fuentes de financiamiento nacional. El sistema se implementó usando tecnologías web de punta que permiten su fácil uso y actualización. En conclusión, la herramienta desarrollada pueda generar nuevas oportunidades de negocios, especialmente para emprendedores desorientados en la materia, y no solo en el territorio nacional, sino también internacional.

Palabras clave: modelos de negocio; planes de negocio; software de gestión; soporte al emprendimiento

\section{Development of a web software for business plan management}

\begin{abstract}
This research article presents the design and development of a web system to support the generation of business models and plans. The system is aimed at new and established entrepreneurs from small and micro enterprises. The main goal of the system is to improve the odds of entrepreneurs to access and obtain financial aid when applying to national funding. The system provides a series of support tools such as expert coaching and template management. The system was developed using current web technologies to facilitate usage and updates. In conclusion, the web tool developed in the present study can be used to generate new business opportunities, especially for new and inexperienced national and international entrepreneurs.
\end{abstract}




\section{INTRODUCCIÓN}

En el año 2017, se identificaron 1.992 .578 emprendedores en Chile (Krell, 2018), de los cuales, el 68,6\% recurre a recursos propios como fuente de financiamiento inicial, el 7,6\% opta por financiamiento privado y sólo el 2,5\% accede a financiamiento público. Estas cifras pueden implicar la existencia de brechas de acceso al financiamiento para el segmento de microempresas, según el Ministerio de Economía de Chile (Krell, 2018). Por otro lado, según el estudio GEM 2015 (Amoros et al., 2015), existe temor al fracaso de crear un nuevo negocio por parte de los emprendedores nacionales entre 18 y 64 años de edad, donde el 20,9\% se encuentran en etapas iniciales del emprendimiento y el $22,2 \%$ con algún tipo de negocio ya constituido.

En la actualidad, el problema clave de las empresas establecidas para su crecimiento, es que no pueden costear las últimas tecnologías para incorporarlas en los procesos de su negocio. Además, al $81 \%$ de las nuevas empresas, les resulta difícil ingresar a nuevos mercados (Amoros et al., 2015), con el fin de fortalecer y elevar los ingresos del negocio. Por lo expuesto, surge la necesidad de buscar financiamiento de fondos públicos o privados, con tal de obtener el equipamiento que logre dar ese impulso que cada emprendedor requiere para fortalecer y potenciar aún más su negocio. Por ejemplo, en la primera convocatoria para el Capital Semilla Corfo año 2018, postularon 1.324 proyectos de los cuales 523 fueron rechazados y 379 no pertinentes, quedando 422 proyectos pre aprobados para las siguientes etapas (CORFO, 2018). Una forma para disminuir la brecha existente entre los emprendimientos que postulan versus aquellos que se adjudican fondos y los temores de los emprendedores al fracaso, es aportar con el conocimiento en la generación de planes de negocios que son requeridos por distintas fuentes financiadoras $y$, de esta forma, aumentar la probabilidad de que los emprendedores puedan adjudicarse estos fondos y lograr explotar su idea de negocio, como también fortalecerlos en el caso de los negocios establecidos.

Un plan de negocio, tiene como objetivo principal analizar y planificar la estrategia de un negocio, basándose en la siguiente estructura común por los fondos nacionales públicos y privados: Resumen ejecutivo, Definición del negocio, Estudio de mercado, Estudio técnico, Organización, Estudio de la inversión y financiamiento, Estudio de los ingresos y egresos, y Evaluación del proyecto. El conocimiento de cada punto correspondiente a la estructura mencionada, es vital para la elaboración de un plan de negocio. Si se desconoce alguno de ellos, se genera frustración y desmotivación en las personas que tienen una idea, pero poca orientación y habilidad para describir correctamente el negocio a realizar, cometiendo errores comunes como utilizar demasiadas palabras, no identificar la oportunidad de mercado, no describir claramente cuál es el negocio a abordar y no dejar claro sus términos. Así, en base a los datos aportados por (CORFO, 2018) se puede inferir que las personas pierden el interés en participar en diversos programas o concursos, producto de la complejidad que ven al realizar un plan de negocios o bien, lo realizan pero son rechazados por las entidades financieras, impactando directamente en la idea inicial y dándole fin a una posible oportunidad comercial.

Así mismo, se puede inferir que lamentablemente, existe una gran cantidad de proyectos rechazados, debido al desconocimiento o falta de orientación al momento de realizar un plan de negocio (CORFO, 2018). Por esto, se busca disminuir la brecha existente a través de un sistema orientado a la web que apoye en su totalidad a los emprendedores que quieran realizar su plan de negocio con una alta probabilidad de ser beneficiario. Se aplicaron tecnologías modernas para una mayor interacción entre usuario y sistema, como también un mecanismo para contar con el apoyo de profesionales que estarán a cargo de emprendedores, cumpliendo la función de validar los documentos que podrán ser presentados a distintos fondos concursables, ya sean públicos o privados, pero esta vez, con una mayor probabilidad de obtener el financiamiento. En la Figura 1, se muestra la relación existente entre el problema, solución y aspectos tecnológicos involucrados en el desarrollo. En el primer nivel, se encuentra la problemática basada en el desconocimiento de las secciones de un plan de negocio, y cómo se podría explotar una idea en una oportunidad comercial. El segundo nivel, hace referencia a la solución, la cual consiste en una plataforma web que pueda elaborar y gestionar planes de negocios, logrando, por un lado, la rapidez de entendimiento a través de ejemplos, y, por otro lado, evitar la pérdida de tiempo al consultar en otras fuentes de información. La plataforma web fue concebida en tres niveles o capas, teniendo la presentación como capa superior que estará encargada de la interacción entre sistema y usuario a través de una interfaz gráfica intuitiva, es decir, entendible y fácil de usar. La capa de presentación se comunica únicamente con la capa intermedia, donde se establecen todas las reglas y funcionalidades que deben cumplirse. Esta capa se comunica con la de presentación para recibir las solicitudes y presentar los resultados. La última capa, permite el acceso a datos para solicitar al gestor de bases de datos almacenar o recuperar información.

\section{OTROS ANTECEDENTES}

Existen varías definiciones, pero la que más se usa es que emprender es una forma de enfrentarse al mundo, enfrentarse a desafíos con una gran incertidumbre, sin tener la certeza de qué pasará el día de mañana pese a los riesgos considerados. Por esto mismo, el verdadero emprendedor es aquel a quien lo incierto procura un especial placer (Trías de Bes, 2007). 


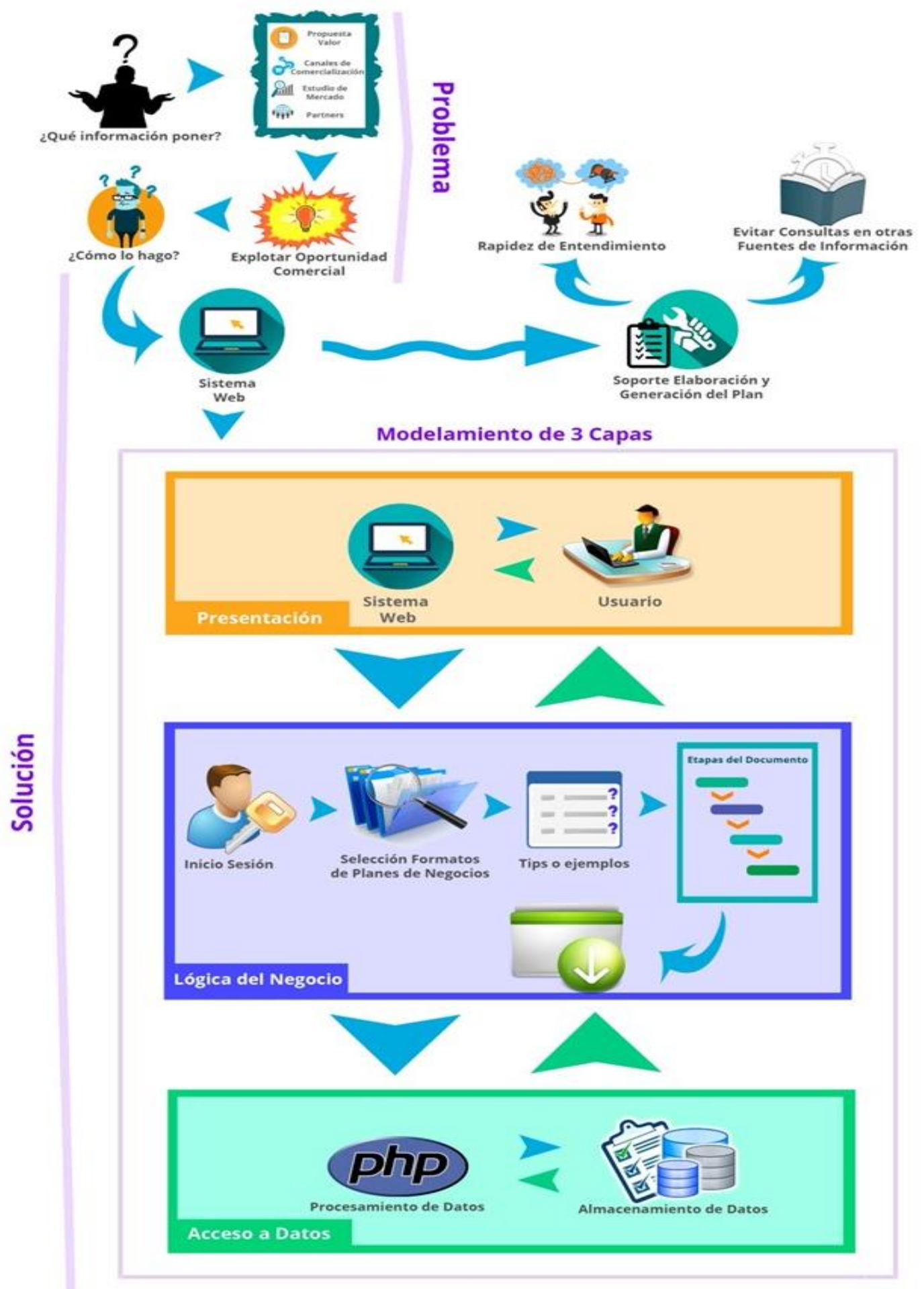

Fig. 1: Diagrama del problema, solución y aspectos tecnológicos.

El proceso de emprender comienza con la concepción de una idea, la cual debe resolver una necesidad real, para posteriormente plasmarla en un documento que permita planificar dicha idea en un proyecto más estructurado, considerando metas, objetivos a corto, mediano y largo plazo, herramientas a utilizar, recursos humanos, competidores, quiénes serán los clientes, entre otras descripciones que van a dar forma a un plan de negocio. Posteriormente, para poder hacer la ejecución del plan, debe ser validado por un equipo profesional, para luego hacer las respectivas inversiones, considerando los riesgos que pueden ocurrir en un tiempo determinado. Ahora bien, para poder hacer crecer el emprendimiento y saltar a otro nivel, se debe formalizar y crear la empresa. Una vez que se obtiene el crecimiento de la empresa, deben existir mejoras continuas, ya sean en conocimientos, infraestructuras, planes de marketing y ventas, entre otras que van a permitir obtener nuevas herramientas para fortalecer la empresa en el rubro que pertenece. Esto último se logra con un plan de negocio. Para poder generar un plan de negocio, es necesario el uso de un método que permita visualizar el modelo de negocio. 


\section{Método para generar un modelo de negocio}

Unas de las herramientas más prácticas y utilizadas a nivel mundial, es el CANVAS, creado por los autores Alexander Osterwalder e Yves Pigneur, quienes definen un modelo de negocio que describe las bases sobre la que una empresa crea, proporciona y capta valor (Osterwalder y Pigneur, 2010). Este describe de manera lógica la forma en que las organizaciones crean, entregan y capturan valor a través de la división de un proyecto en nueve módulos que interactúan entre sí, con el fin de obtener como resultado diferentes formas de hacer rentable la empresa. Los módulos son: Segmentos de mercado, Propuestas de valor, Canales de contacto con los clientes, Relación con el cliente, Fuentes de ingresos, Recursos clave, Actividades clave, Asociaciones clave y Estructura de costos (Ferreira-Herrera, 2015). Contando con estos elementos, es posible generar el plan de negocios.

\section{Plan de negocio}

Un plan de negocio es vital para iniciar un emprendimiento, como también para potenciar empresas establecidas que necesitan de recursos claves que no están al alcance de sus utilidades. Pero, ¿por qué es importante? Es de suma importancia, debido a que con un plan de negocio es posible presentar el emprendimiento a inversionistas, a fuentes de financiamientos públicos o privados, y de esta forma, obtener esos recursos que harán crecer el negocio, teniendo nuevas tecnologías en los procesos de producción para lograr una mayor competitividad en el mercado. Un plan de negocio es un documento donde el emprendedor o empresario plasma su reflexión estratégica y detalla toda información relacionada con su empresa o negocio. Se define según Thomsen (Thomsen y Gerard, 2009), "El plan de negocio recoge todos los elementos de su información comercial y su experiencia de vida en general que sean pertinentes para su negocio. Contribuye a un inicio más ordenado - en beneficio del emprendedor y su familia, red personal, consultores, y fuentes de financiamiento" (página 7 , párrafo 2).

Para una adecuada elaboración del plan de negocio, se puede seguir el proceso que se muestra en la Figura 2, en donde se aprecia que, a partir de una Idea de Negocio, se debe realizar una evaluación previa de su potencial viabilidad antes de pasar a desarrollar un análisis estratégico que permitirá definir el Modelo de Negocio de la empresa. A su vez, el modelo planteado es la base para formular el plan de negocio que, de presentar dificultades, puede indicar, asimismo, su revisión.

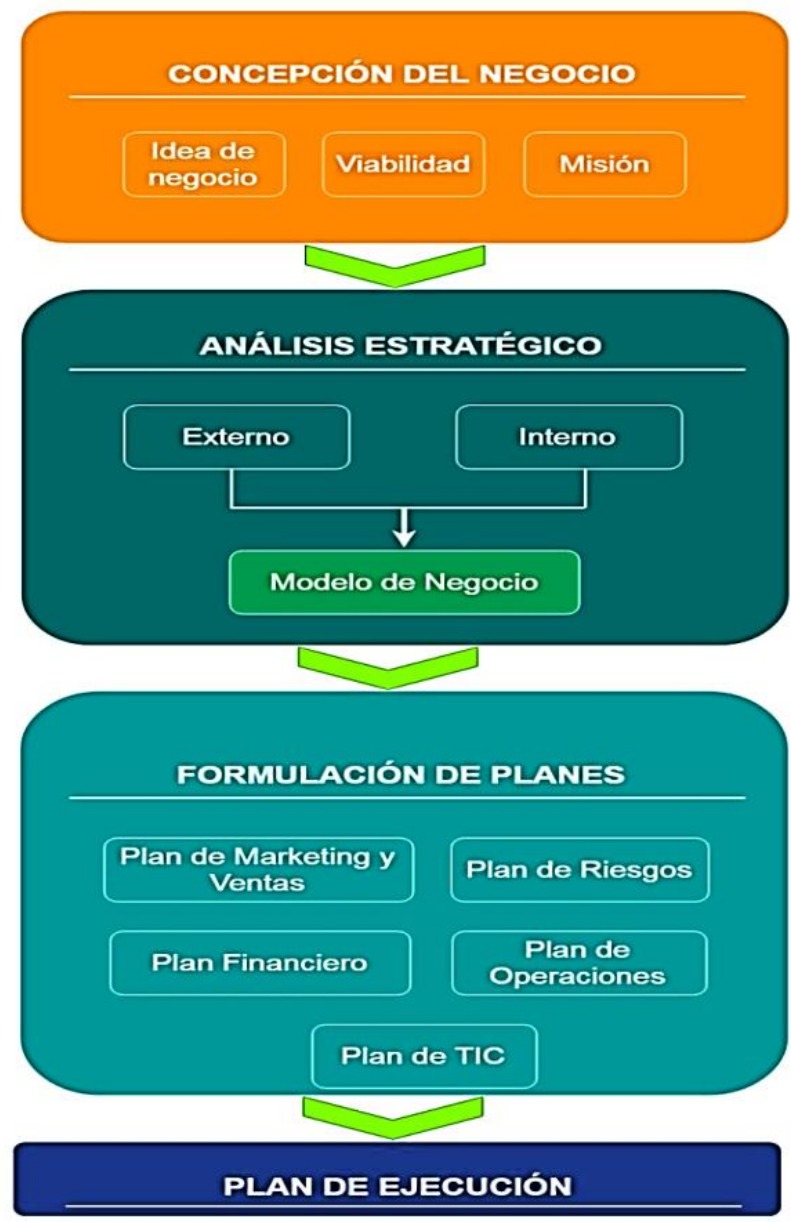

Fig. 2: Diagrama del proceso para la elaboración de un plan de negocios 
Por último, se debe plantear cómo poner en marcha los Planes Definidos para garantizar su éxito. Además, es muy probable que el desarrollo de una etapa, aporte información que aconseje adaptar etapas anteriores (por ejemplo, al realizar el análisis externo, se puede constatar que la Misión planteada no es la más apropiada para aprovechar las oportunidades que ofrece el mercado). Con este proceso terminado, es posible optar a diversas fuentes de financiamiento, como las que se detallarán a continuación.

\section{Fuentes de financiamientos nacional y sus requerimientos}

La necesidad de creación de nuevas fuentes de trabajo se ha vuelto una preocupación para los países sudamericanos, especialmente en lo que tiene que ver con crear y potenciar la generación de emprendimientos. En este contexto, Chile tampoco está exento de esta preocupación. Existen distintos fondos concursables, tales como: Capital Abeja, Capital Semilla Emprende, Crece Multisectorial, Semilla CORFO, Yo Emprendo Semilla, Fondo del Adulto Mayor, Youth ActionNet, Premio Jump Chile, The S Factory (Start Up Chile), Seed (Start Up Chile), y Seguimiento (Start Up Chile). En (Ocampo et al., 2018), pueden apreciarse datos como institución que lo ofrece, como también una breve descripción y sus principales requerimientos. Como se mencionó anteriormente, en la actualidad muchos de los fondos se pierden por la falta de la generación de planes de negocios que cumplan con los requerimientos de dichos fondos, por lo cual se hace necesario contar con una herramienta adecuada para la realidad local. A pesar de la existencia de herramientas, estas no constan con los debidos elementos, como se analizará en la siguiente sección.

\section{Trabajos relacionados}

Si bien existen herramientas que apoyan parte del proceso de gestión de planes de negocios, no todas aplican tecnologías modernas para su implementación, y tampoco cuentan con un usuario profesional a cargo de validar planes de negocios a emprendedores (al que se denomina "coach"). Algunos ejemplos de trabajos relacionados se presentan a continuación y, posteriormente, se muestra una comparativa entre ellas y el sistema que se presenta en este artículo.

Metodología para la integración del modelo de negocios CANVAS y un mapa de ruta tecnológico: En este artículo, desarrollado por Toro-Jarrin (Toro-Jarrin et al., 2016), se utiliza la metodología CANVAS para evaluar una idea mediante los nueve componentes que lo constituye, con el fin de concluir si dicha idea puede convertirse en un negocio. Una vez desarrollado el modelo de negocio y con una evaluación positiva, se debe formalizar y plasmar la idea de negocio base en un plan de negocio a través de una estructura que se divide en dos secciones. La primera es "Business Model Canvas", en donde se incluye la presentación, proceso del modelo y ejemplo. La segunda es "Modelo de negocio y modelo de operaciones", teniendo un resumen ejecutivo, descripción del proyecto, mercado, valor diferencial y ventajas competitivas, equipo, estrategia y cadena de valor, análisis de la situación (DAFO), momento actual, aspectos económicos y financieros, riesgos y otros.

Nueva metodología CANVAS. Innovación en Modelos de Negocio: Metodología Lean Canvas en un Start-up de Base Tecnológica. En este artículo desarrollado por Diego Fino Garzón (Fino-Garzón, 2013) presenta una nueva versión de la metodología CANVAS, creada por Ash Maurya (Maurya, 2016) y enfocada a las Start-ups. Las Startups se definen como "Empresas de reciente creación y con grandes posibilidades de crecimiento. Éstas son compañías fundadas con un claro espíritu emprendedor y suelen estar asociadas a la innovación y desarrollo de nuevas tecnologías". Si bien la metodología CANVAS es un referente para emprendedores y grandes empresas, la adaptación de Ash Maurya conocida como Lean Canvas, hace referencia a identificar las áreas de mayor riesgo cuando un emprendedor está tratando de decidir si tiene realmente una oportunidad de negocio basada en las tecnologías de información y comunicación.

Herramienta de Software para Preparar Planes de Negocios (UnBizPlanner): En este artículo desarrollado por Cifuentes (Cifuentes et al., 2008), se presenta una herramienta software para la generación de un plan de negocio, enfocada para pequeñas y medianas empresas que deseen buscar financiamiento, socios o inversionistas, en Colombia.

Herramienta E.S. Plan de empresa. Es una herramienta para generar un plan de negocio ya definido, además cuenta con textos de ayuda para cada sección de la estructura de contenido del plan. También se encuentra disponible para usuarios con sistema operativo Windows o Mac. Sin embargo, no es una herramienta orientada a la web que permita a los usuarios trabajar en la plataforma desde distintos lugares del mundo. (E.S. Plan, 2018).

En la Tabla 1, se muestra la comparativa entre los trabajos relacionados y el sistema desarrollado. Se destaca que si bien apoyan parte del proceso de gestión de planes de negocios, no todas aplican tecnologías modernas para su implementación y tampoco cuentan con un coach para validar planes de negocios a emprendedores. Algunas no describen la arquitectura de diseño. 
Tabla 1: Estudios y herramientas existentes VS herramienta propuesta

\begin{tabular}{|l|l|l|l|l|l|l|l|}
\hline $\begin{array}{l}\text { Trabajo } \\
\text { relacionado }\end{array}$ & $\begin{array}{l}\text { Dispone de } \\
\text { herramienta }\end{array}$ & $\begin{array}{l}\text { Tecnologías } \\
\text { modernas }\end{array}$ & $\begin{array}{l}\text { Para la } \\
\text { web }\end{array}$ & $\begin{array}{l}\text { Cubre todo } \\
\text { el proceso }\end{array}$ & $\begin{array}{l}\text { Sigue un } \\
\text { método }\end{array}$ & $\begin{array}{l}\text { Arquitectura } \\
\text { diseño software }\end{array}$ & $\begin{array}{l}\text { Validación } \\
\text { profesional }\end{array}$ \\
\hline $\begin{array}{l}\text { Toro-Jarrin } \\
\text { et al., 2016 }\end{array}$ & No & No aplica & No aplica & No & $\mathrm{Si}$ & No aplica & No aplica \\
\hline $\begin{array}{l}\text { Fino-Garzón, } \\
2013\end{array}$ & $\mathrm{No}$ & No aplica & No aplica & No & $\mathrm{Si}$ & No aplica & No aplica \\
\hline $\begin{array}{l}\text { Cifuentes et } \\
\text { al., 2008 }\end{array}$ & $\mathrm{Si}$ & $\mathrm{No}$ & $\mathrm{No}$ & $\mathrm{No}$ & $\mathrm{Si}$ & $\begin{array}{l}\text { No hay } \\
\text { información }\end{array}$ & No \\
\hline $\begin{array}{l}\text { E.S. Plan, } \\
\text { 2018 }\end{array}$ & $\mathrm{Si}$ & $\mathrm{No}$ & $\mathrm{No}$ & $\mathrm{No}$ & $\mathrm{No}$ & $\begin{array}{l}\text { No hay } \\
\text { información }\end{array}$ & No \\
\hline $\begin{array}{l}\text { Sistema } \\
\text { Desarrollado }\end{array}$ & $\mathrm{Si}$ & $\mathrm{Si}$ & $\mathrm{Si}$ & $\mathrm{Si}$ & $\mathrm{Si}$ & $\begin{array}{l}\text { Modelo Vista } \\
\text { Controlador }\end{array}$ & $\mathrm{Si}$ \\
\hline
\end{tabular}

\section{MATERIALES Y MÉTODOS}

En el presente trabajo se utilizó investigación aplicada en virtud de que se presenta una solución tecnológica a un problema concreto, como los problemas abordados en los trabajos de Cabarcas et al. (2015), Coca et al. (2016), Alarcón-Aldana et al. (2016), Gómez et al. (2016), Mamani et al. (2017), Quintero et al. (2017) y Vidal et al. (2017). Para una descripción detallada del método y los materiales, utilizados en el desarrollo de la herramienta de software que se describe en este artículo, pueden revisarse en Castro-Rivera, et al. (2020). Esto es porque en ambos trabajos, se utilizaron los mismos métodos y herramientas de desarrollo, ya que son materiales ya estudiados y generalizados, en la Ingeniería de Software.

\section{RESULTADOS}

Los resultados se presentan y discuten en varias subsecciones: proceso de negocio, arquitectura del sistema, casos de usos, diagrama relacional, implementación y pruebas.

\section{Proceso de negocio}

En primer lugar, se modeló el proceso de negocio. En la Figura 3, se puede apreciar el modelo realizado donde actúan los distintos usuarios. Se puede apreciar que el administrador registra usuarios para, posteriormente, hacer las asignaciones entre emprendedores y coaches. Luego, deberá crear un modelo de negocio, como también un plan de negocio, para poder realizar la estructura de contenido de estos documentos, por lo que deberá cambiar el estado de visibilidad con el fin de que puedan ser vistos por emprendedores y coaches.

Por otro lado, el emprendedor podrá desarrollar el modelo de negocio publicado en el sistema. Una vez terminado, deberá esperar a que su modelo sea validado por su coach, permitiendo visualizar y desarrollar los planes de negocios disponibles, los cuales también serán validados por su coach. Una vez que este último realice la validación de su plan, el emprendedor podrá hacer la descargar del documento en formato PDF, listo para ser impreso o enviado a alguna entidad financiadora. Por último, el coach podrá ver a todos los emprendedores que les fueron asignados junto a sus documentos, ya sea un modelo o planes de negocios, para poder evaluarlos y validarlos. Cabe destacar que el proceso exige que un emprendedor termine su modelo de negocio para que sea validado por el coach, de lo contrario no podrá trabajar en ningún otro plan de negocio disponible.

\section{Arquitectura del sistema}

Para concretar un marco de referencia de la construcción del sistema, se define un conjunto de componentes y las interacciones que tienen éstos, para llevar a cabo alguna función específica. Este conjunto de componentes a nivel de sistema se conoce como arquitectura del sistema. Al igual que los métodos y herramientas para el desarrollo del software, la descripción detallada de la arquitectura, puede revisarse en Castro (Castro-Rivera, et al., 2020), por la misma razón expuesta en la sección Materiales y Métodos.

\section{Casos de Usos}

A continuación, se describen los principales casos de uso para el desarrollo del proyecto, representando las funciones provistas por el sistema a distintos tipos de actores. 


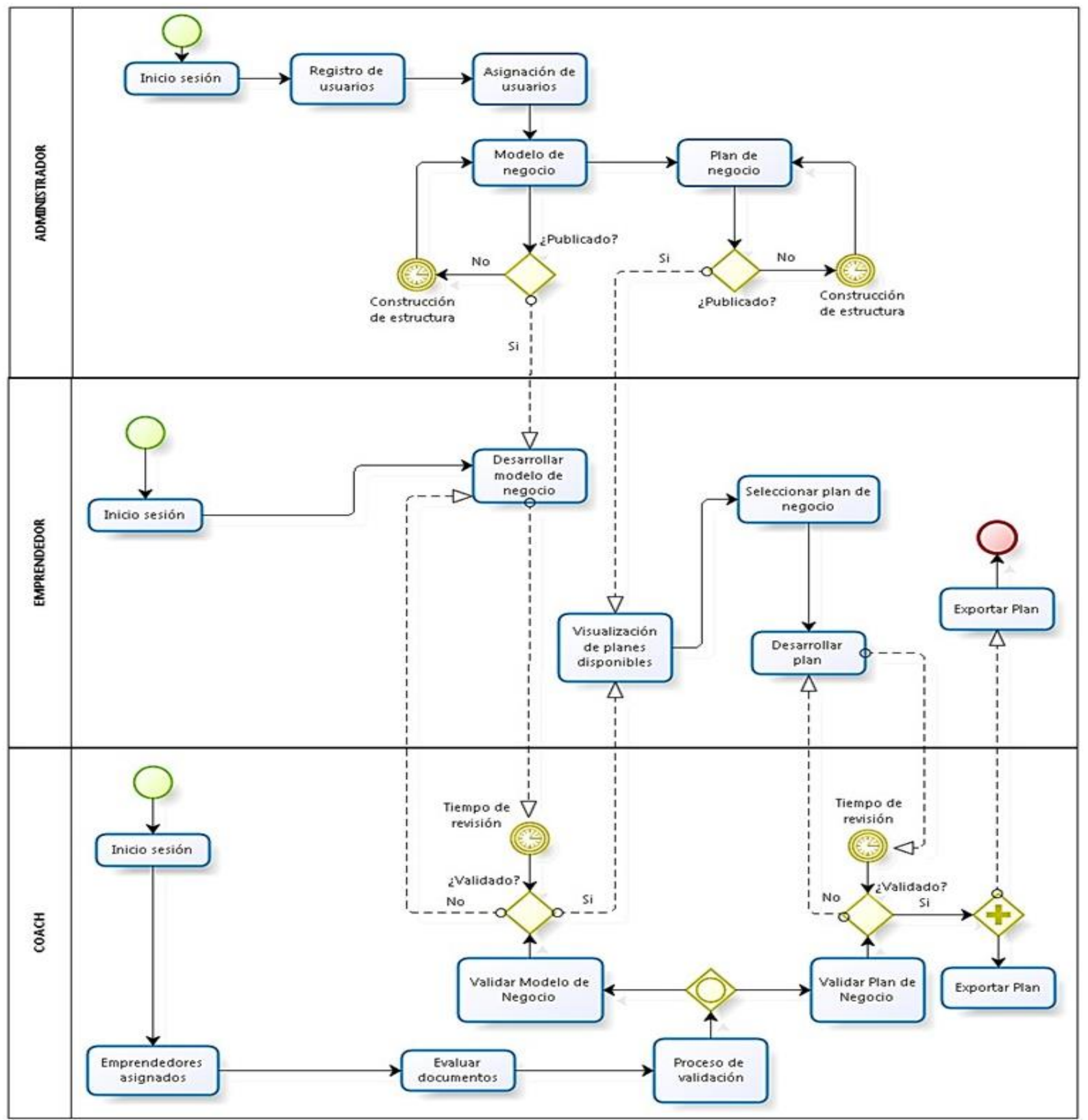

Fig. 3: Diagrama del modelo de proceso de negocio.

Caso de uso Gestionar Usuario: Muestra la interacción que tiene el administrador y el coach para gestionar usuarios. El administrador puede modificar, eliminar o crear un nuevo registro. En este último caso, puede también crear un perfil del usuario previamente registrado en el sistema. Además, puede realizar asignaciones entre usuarios coach y usuarios emprendedores, con el fin de que cada coach esté a cargo de uno o más emprendedores. Por otro lado, el coach puede consultar y ver a todos los emprendedores que les fueron asignados por el administrador, con la finalidad de ver cada documento desarrollado por un emprendedor, ya sean modelos de negocios o planes de negocios.

Caso de uso Gestionar Modelos de Negocios: Muestra la interacción que tienen los distintos tipos de usuarios para gestionar modelos de negocio (MN). El administrador puede consultar los modelos de negocio existentes para seleccionar uno y poder administrar secciones, atributos e ítems con la finalidad de crear la estructura de contenido del documento. Por otro lado, el coach puede visualizar los modelos de negocios que le pertenecen a emprendedores que le fueron asignados $\mathrm{y}$, de esta forma validarlos, para que pueda ser exportado en formato PDF. Además, el administrador será capaz de crear modelos plantillas para modelos y planes de negocios específicos, para ser presentadas a fuentes de financiamiento nacional, como las mencionadas en secciones anteriores, facilitando así la postulación por parte del emprendedor. Finalmente, el emprendedor desarrolla o rellena todos los modelos de negocios que se encuentren creados y visibles en el sistema. Al terminar un modelo de negocio, también podrá descargar el documento mientras éste se encuentre validado por el coach. 
Caso de uso Administrar Modelos de Negocios: En este caso uso, se aprecia la interacción que tiene el administrador, donde puede crear un nuevo modelo de negocio con la información general, como también modificar o eliminar dicha información. Cabe destacar que la eliminación de un modelo de negocio implica también en eliminar la estructura de contenido que posee, mientras dicho documento no esté siendo utilizado por un usuario emprendedor.

Caso de uso Gestionar Planes de Negocios: Muestra la interacción que tienen los distintos tipos de usuarios para gestionar planes de negocios (PN). El administrador puede consultar los planes de negocio existentes para seleccionar uno y poder administrar secciones, atributos e ítems con la finalidad de crear la estructura de contenido del documento. La diferencia del presente caso de uso en comparación a Gestionar Modelos de Negocio, es que el administrador puede clonar atributos que pertenecen a un modelo de negocio con la finalidad de que al momento en que el emprendedor desarrolle un plan de negocio que contenga atributos de su propio modelo, no deba reescribirlos. Por otro lado, el coach puede visualizar los planes de negocios que le pertenecen a emprendedores que le fueron asignados, y de esta forma validarlos para que puedan ser exportados a formato PDF. Finalmente, el emprendedor desarrolla o rellena todos los planes de negocios que se encuentren creados y visibles en el sistema. Al terminar un plan de negocio, también podrá descargar el documento mientras éste se encuentre validado por el coach.

Caso de uso Administrar Planes de Negocios: En este caso uso, se aprecia la interacción que tiene el administrador con la administración de planes de negocio, donde puede crear un nuevo plan de negocio con la información general, como también modificar o eliminar dicha información. Cabe destacar que la eliminación de un plan de negocio implica también en eliminar la estructura de contenido que posee, mientras dicho documento no esté siendo utilizado por un usuario emprendedor.

\section{Diagrama Relacional}

En la Figura 4, se puede apreciar el diagrama lógico relacional, para el desarrollo del sistema en donde se muestran once entidades o tablas que fueron utilizadas para la persistencia de datos.

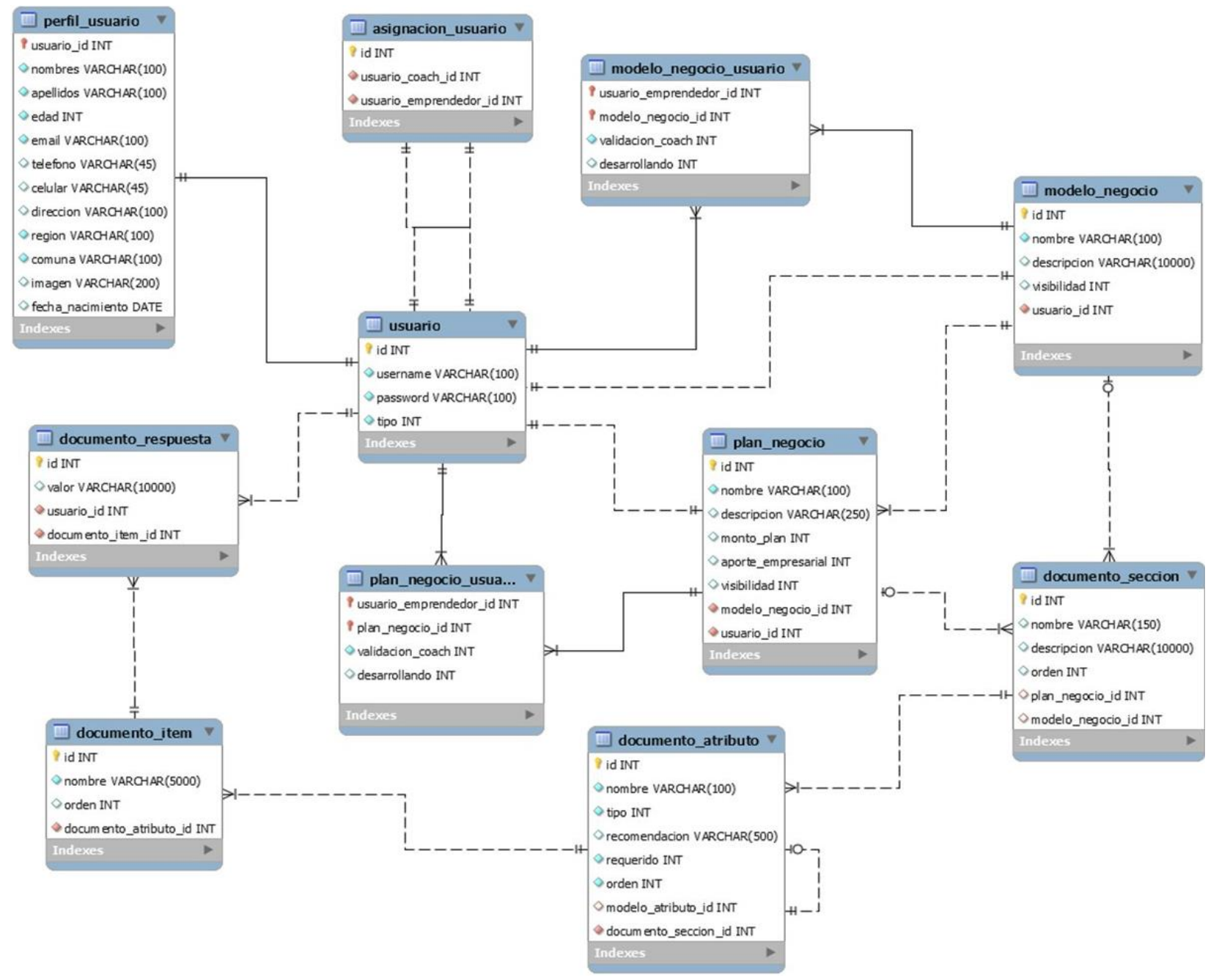

Fig. 4: Diagrama del modelo lógico relacional. 


\section{Implementación}

Tanto el desarrollo, incluidas las pruebas, como la puesta in-situ (operación) fueron realizadas en las instalaciones de la empresa Kuvemar (2020). La implementación consideró tres perfiles de usuario: administrador, coach y el emprendedor.

Vista principal. En la Figura 5, se puede apreciar la vista principal desde la cual los distintos perfiles pueden acceder a sus respectivos servicios.

\section{Sistema Inicio Iniciar Sesión}

\section{Plataforma Web para la Generación de Planes de Negocios}

Adquiere mejoras para tu emprendimiento a través de postulaciones a financiamientos publicos o privados.

\section{¡Explota o potencia tu idea!}

Si tienes una idea de negocio, la podrás explotar con la ayuda de esta plataforma web, ya que ayudará en la construcción de un plan de negocio para presentarlo a distintas fuentes de financiamiento (públicas o privadas).

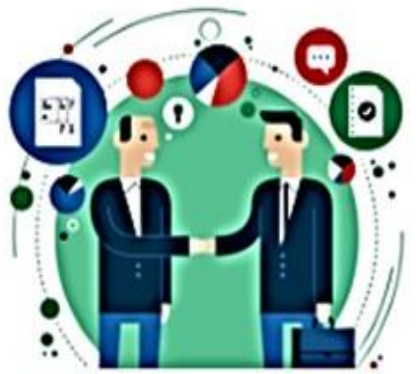

\section{Asesoría Experta}

Nuestro objetivo es generar impacto en la adquisición de proyectos para emprendedores 0 pequeños empresarios a través de profesionales expertos que validarán tu plan de negocio para que tengas grandes probabilidades de potenciar tu negocio.

\section{¿COMENCEMOS?}
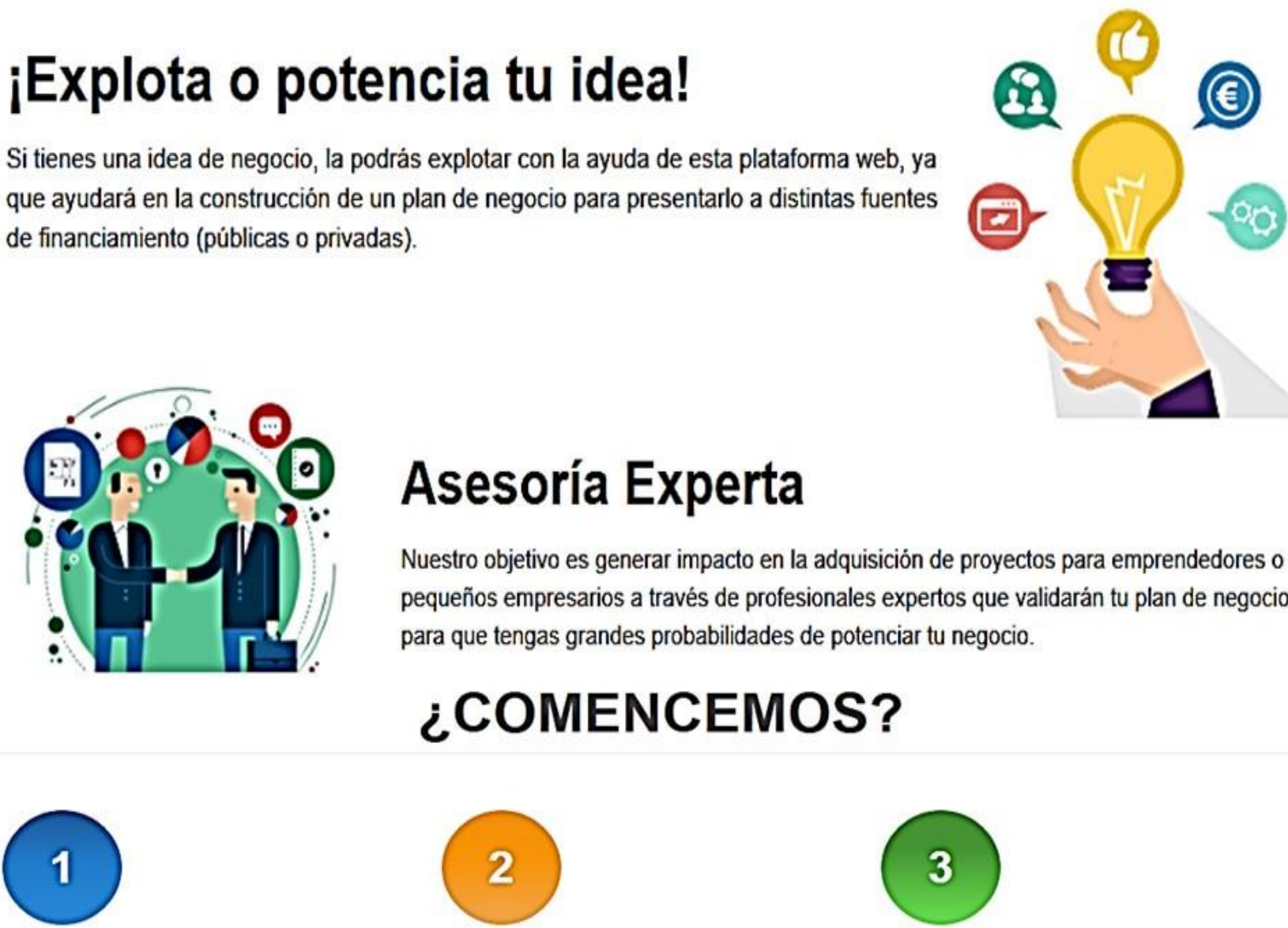

\section{Modelo de Negocio Plan de Negocio}

Genera el modelo de negocio base de tu empresa a través de la metodología Canvas, el podrás seleccionar cualquier plan de negocio cual será validado por un experto del área. Desarrollado y validado tu modelo de negocio, que pertenecen a distintos fondos concursables. En esta etapa, nuestros expertos fuentes financiadoras. también te apoyarán.

\section{3}

\section{Exporta tu plan}

Cuando tengas el modelo o plan de negocio validado, podrás realizar la descarga de estos documentos para presentarlos a las distintas 
Vista Administrador. En la Figura 6a, se presentan la barra de navegación diseñada para el administrador, pudiendo acceder a los servicios para gestionar su perfil, los usuarios (emprendedores, coach), modelo de negocios, planes de negocios y cierre de sesión. En la Figura 6b, se presenta la "Vista Planes de Negocios", la que permite administrador mostrar todos los planes de negocios creados en el sistema. Además tiene la opción de crear o modificar una estructurar existente, como también deshabilitar o habilitar un plan de negocio, esto sirve para cambiar la visibilidad de dicho documento, es decir, si se encuentra habilitado, todos los usuarios emprendedores y coaches podrán ver y trabajar en los planes que se encuentren con este estado. En la Figura 6c, se puede apreciar la "Vista Estructurar Plan de Negocio". Al seleccionar el botón "Estructurar" (figura 6b) un plan de negocio, muestra la información general del plan, como también los botones para visualizar y descargar el plan de negocio. Además, el usuario administrador puede comenzar a crear la estructura del documento, iniciando con las secciones. En la Figura 6d, se presenta la "Vista para crear secciones". Al seleccionar el botón para crear una nueva sección (figura 6c), el sistema despliega un formulario flotante para poder realizar dicha acción. En la Figura 6e, se muestra como una vez que el administrador completa el formulario y crea la nueva sección, el sistema re direcciona a la vista estructurar plan con el nuevo registro, permitiendo al administrador ver, modificar o eliminar secciones existentes. En la Figura 6f, se puede apreciar la "Vista para crear atributos", donde se puede ver, modificar o eliminar todos los atributos que corresponden a una sección, como también crear nuevos atributos. En primer lugar, el sistema muestra una lista con todos los atributos para la sección "Identificación del Proyecto". En segundo lugar, si el administrador desea crear un nuevo atributo, el sistema despliega un formulario flotante para que el administrador lo pueda rellenar y crear (figura $6 \mathrm{~g}$ ). En la Figura 6h, se muestra la "Vista para crear ítems", donde se puede ver los ítems que le corresponde a un atributo. En este caso, el atributo de nombre "Sector Económico" y de tipo selección, posee tres ítems que pueden ser modificados o eliminados. Si el administrador desea agregar un nuevo ítem, el sistema despliega un formulario flotante para que el administrador ingrese el nombre del nuevo ítem, tal como se muestra en la (figura 6i).

\begin{tabular}{|c|c|cccc|}
\hline Sistema & Inicio & Mi Perfil & Usuarios - Modelo de Negocio $\quad$ Planes de Negocio Cerrar Sesión (admin) \\
\hline
\end{tabular}

Fig. 6a. Barra de navegación diseñada para el administrador

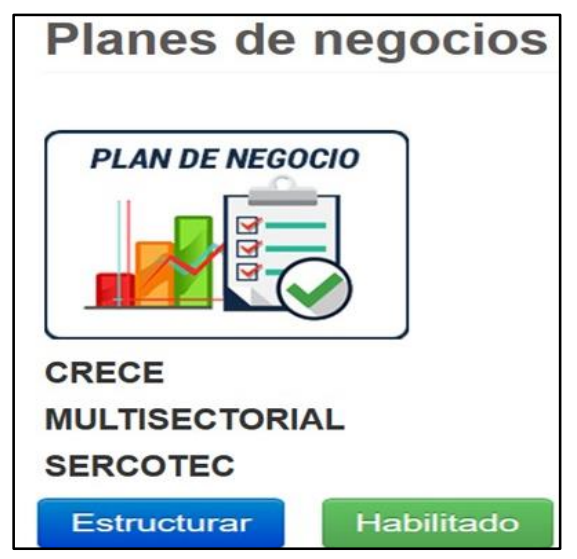

Fig. 6b. Vista opción Planes de Negocios

\begin{tabular}{|c|c|c|c|c|}
\hline \multicolumn{4}{|c|}{ Estructura Plan - CRECE MULTISECTORIAL SERCOTEC } & \multirow[t]{2}{*}{ OVisualizar } \\
\hline \multicolumn{4}{|c|}{4 Descargar } & \\
\hline \multicolumn{2}{|c|}{ Nombre Plan: } & \multicolumn{2}{|c|}{ CRECE MULTISECTORIAL SERCOTEC } & \\
\hline \multicolumn{5}{|c|}{ Descripción: } \\
\hline \multicolumn{2}{|c|}{ Monto concursable: } & \multicolumn{2}{|c|}{6500000} & \\
\hline \multicolumn{2}{|c|}{ Aporte empresarial: } & \multicolumn{2}{|c|}{$30 \%$ del valor neto } & \\
\hline \multicolumn{2}{|c|}{ Creado por: } & \multicolumn{2}{|c|}{ Marco Andrés Villalobos Abarca } & \\
\hline \multicolumn{5}{|c|}{ Secciones } \\
\hline Orden & Nombre" & Descripcion" & Atributos & Operaciones \\
\hline
\end{tabular}

Fig. 6c. Vista opción Estructurar Plan de Negocio 


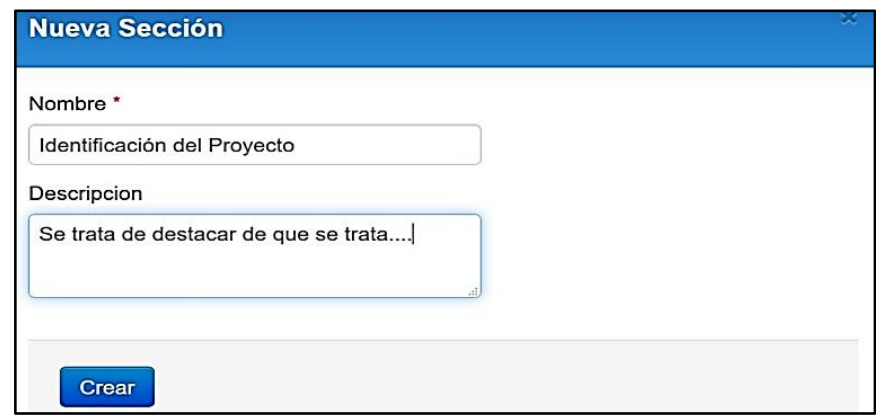

Fig. 6d. Vista opción Crear Secciones

\begin{tabular}{|c|c|c|c|c|}
\hline \multicolumn{5}{|c|}{ Estructura PIan - CRECE MULTISECTORIAL SERCOTEC } \\
\hline \multicolumn{2}{|c|}{ Nombre Plan: } & \multicolumn{3}{|l|}{ CRECE MULTISECTORIAL SERCOTEC } \\
\hline \multicolumn{5}{|c|}{ Descripción: } \\
\hline \multicolumn{2}{|c|}{ Monto concursable: } & \multicolumn{3}{|l|}{6500000} \\
\hline \multicolumn{2}{|c|}{ Aporte empresarial: } & \multicolumn{3}{|l|}{$30 \%$ del valor neto } \\
\hline \multicolumn{2}{|c|}{ Creado por: } & \multicolumn{3}{|l|}{ Marco Andrés Villalobos Abarca } \\
\hline \multicolumn{5}{|c|}{ Secciones } \\
\hline \multirow[t]{2}{*}{ Orden } & Nombre & Descripcion & Atributos & Operaciones \\
\hline & $\begin{array}{l}\text { Identificación del } \\
\text { Proyecto }\end{array}$ & Se trata de destacar de que se trata.... & $\bullet$ & ，音 \\
\hline
\end{tabular}

Fig. 6e. Vista opción Ver, Modificar o Eliminar Secciones Existentes

\begin{tabular}{|c|c|c|c|c|}
\hline \multicolumn{5}{|c|}{ Estructura de la Sección - Identificación del Proyecto } \\
\hline \multirow{2}{*}{$\begin{array}{l}\text { Atributos } \\
\text { Orden }\end{array}$} & \multirow[b]{2}{*}{ Nombre } & \multirow[b]{2}{*}{ Recomendación } & \multirow[b]{2}{*}{ Items } & $\odot$ \\
\hline & & & & \\
\hline 1 & Nombre del Proyecto & Debe ser representativo de los objetivos del proyecto & & 甾 \\
\hline 1 & Sector Económico & $\begin{array}{l}\text { Debe representar fielmente el secotor, por ejemplo: pesquero, agricola, } \\
\text { minero, etc. }\end{array}$ & 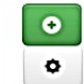 & 息 \\
\hline 1 & Ámbito & Ingeniería & & 自 \\
\hline
\end{tabular}

Fig. 6f. Vista opción Vista para Crear Atributos

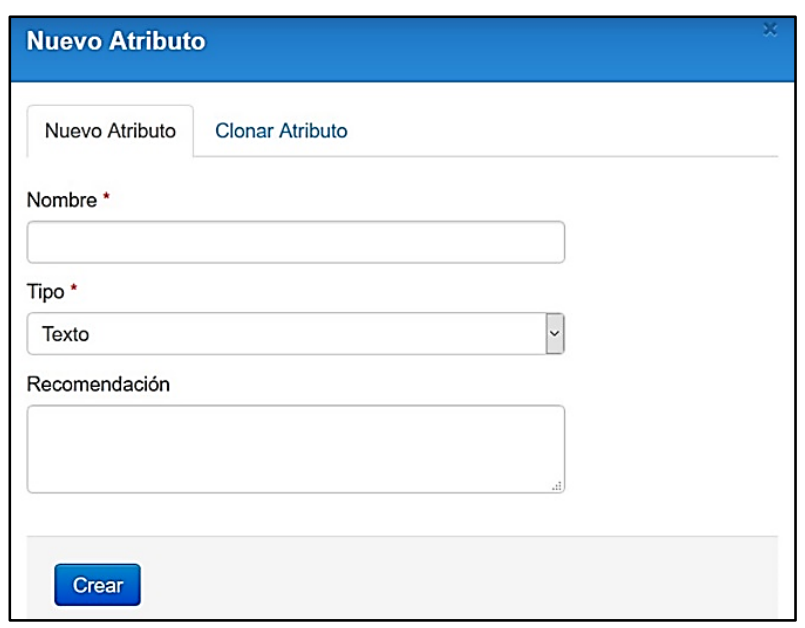

Fig. 6g. Vista opción Crear un Nuevo Atributo 


\begin{tabular}{|c|c|c|c|}
\hline \multicolumn{2}{|c|}{ Atributo - Sector Económico } & & \\
\hline \multirow{2}{*}{$\begin{array}{l}\text { Item } \\
\text { Orden }\end{array}$} & & & $\odot$ \\
\hline & Nombre & & \\
\hline 1 & Minero & \multicolumn{2}{|l|}{ 八畐 } \\
\hline 2 & Agricola & \multicolumn{2}{|l|}{ ，畐 } \\
\hline 3 & Pesquero & \multicolumn{2}{|l|}{ 八畐 } \\
\hline
\end{tabular}

Fig. 6h. Vista opción Crear Ítems

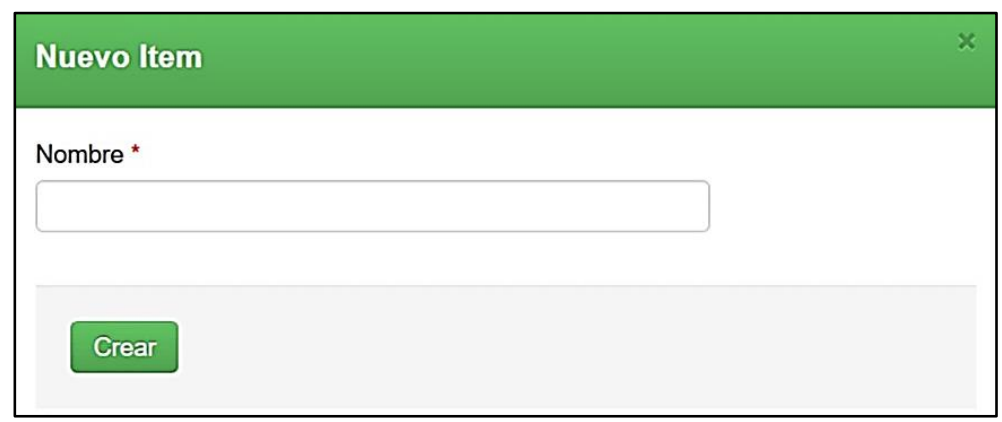

Fig. 6i. Vista opción Ingresar Nombre del Nuevo Ítem

Vista coach. En la Figura 7, se pueden apreciar la barra de navegación diseñada para el coach, pudiendo acceder a los servicios para gestionar su perfil, los emprendedores a su cargo y sus respectivos planes de negocio, y cerrar sesión.

\begin{tabular}{|l|l|lll|}
\hline \hline Sistema & Inicio & Mi Perfil & Emprendedores & Cerrar Sesión (coach) \\
\hline
\end{tabular}

Fig. 7. Barra de navegación diseñada para el coach

Vista emprendedor. En la Figura 8, se puede apreciar la barra de navegación diseñada para los emprendedores, pudiendo acceder a los servicios para gestionar su perfil, CANVAS de su emprendimiento, planes de negocios, y cerrar su sesión.

\begin{tabular}{|l|l|llll|}
\hline \hline Sistema & Inicio & Mi Perfil & CANVAS & Planes de Negocio Cerrar Sesión (emprendedor) \\
\hline
\end{tabular}

Fig. 8. Barra de navegación diseñada para los emprendedores

\section{Pruebas}

El proceso de prueba conlleva la realización de un conjunto de tareas a lo largo del ciclo de vida del sistema. De acuerdo con el estándar IEEE 1012-2016 (Kim et al., 2017), el conjunto mínimo de pruebas que se realizaron fueron las siguientes: a) prueba modular que consiste en la prueba de cada módulo aislado del resto del sistema; b) prueba de integración que se realiza a medida que los diferentes módulos del sistema se integran en el mismo, ya se ha realizado la prueba modular, y se supone que todos los módulos son correctos; el objetivo fundamental de esta prueba es comprobar que las interfaces entre los distintos módulos son correctas; c) prueba del sistema que se realiza cuando se han integrado todos los módulos, y su objetivo es comprobar que el sistema satisface los requisitos del usuario, tanto los funcionales como los no funcionales; y d) prueba de aceptación que se realiza una vez que el sistema se ha implantado en su entorno real de funcionamiento, y su objetivo es demostrar al usuario que el sistema satisface sus necesidades.

Las pruebas arrojaron como resultado que las distintas tecnologías aplicadas en los módulos para el desarrollo del sistema funcionaron correctamente, logrando una eficiente conexión entre las distintas capas de los modelos, vistas y controladores, para tener los resultados vistos anteriormente. Para lograr esto, las funciones desarrolladas 
tuvieron que recibir y enviar información, como también hacer consultas a la base de datos para realizar acciones de guardar u obtener información y, de esta forma, mostrarlas en las vistas que correspondan. Es preciso enfatizar que la función principal del sistema se basa en la generación de la estructura de un documento, ya sea de un modelo o de un plan de negocio, en donde se tuvo que implementar funciones e interfaces que permitan crear la estructura de contenido de forma dinámica y además en orden jerárquico. Por esto, se desarrollaron vistas con múltiples formularios pertenecientes a distintos modelos, los cuales tuvieron un buen resultado, logrando cumplir con las funcionalidades del sistema.

\section{DISCUSIÓN}

La motivación por el emprendimiento permitió desarrollar un sistema que puede generar nuevas oportunidades para todo emprendedor o empresario, aplicando distintas tecnologías informáticas como también metodologías que logren guiarlos en la construcción de un plan de negocio y que pueda apoyar a la consecución de financiamiento externo. Para el sistema, se consideraron fuentes nacionales en relación al emprendimiento, como también las plantillas de planes de negocios que son requeridas por entidades públicas o privadas que financian emprendimientos e ideas de negocios en Chile, entre ellas se encuentran las más importantes CORFO y SERCOTEC, las cuales tienen varios fondos para ser adjudicados. Es importante enfatizar que el programa Startup Chile de CORFO, es la aceleradora líder en Latinoamérica, se posiciona entre las diez mejores a nivel mundial, teniendo como objetivo mantener a Chile como uno de los países de emprendimiento tecnológico e innovación más importantes del mundo.

En base a los resultados obtenidos y a la integración de las tecnologías descritas anteriormente, se desarrolló una herramienta de apoyo para todo emprendedor o empresario que quiera potenciar su negocio, gracias a la nueva adquisición de equipamiento tecnológico, que es posible obtener mediante un correcto desarrollo de un plan de negocio. Es por esto que la herramienta web desarrollada implementó un proceso metodológico en donde inicialmente se debe desarrollar la base de un plan a través de la metodología CANVAS, forzando al emprendedor a (que es indispensable) crear un plan de negocio si aún no logra completar su propio modelo de negocio. Este CANVAS no lo construye solo, lo construye con ayuda del propio sistema, como también de los profesionales que estarán validando sus documentos.

Es importante considerar que el emprendimiento a nivel nacional, no está solamente siendo potenciado a nivel empresarial, sino también a nivel educacional, principalmente en educación superior, como lo discute el trabajo de Herrera-Acuña y Villalobos-Abarca (2018). El sistema consideró en su diseño la posibilidad de ser utilizado por alumnos y profesores de la Universidad de Tarapacá, siendo este el objetivo de un proyecto de investigación en este ámbito (código 8727-18). El acceso a la herramienta se puede realizar desde distintos dispositivos, ya que se encuentra desarrollada con un diseño adaptable. Dicho esto, sería atractivo implementar una aplicación móvil que permita a los emprendedores desarrollar sus documentos, pero lo más importante, recibir notificaciones directas de los profesionales, como también del mismo sistema. Por ejemplo, que el sistema notifique automáticamente cuando un emprendedor tenga documentos incompletos, recordando que debe finalizarlos, como también recibir notificaciones cuando un profesional haya validado sus documentos.

\section{CONCLUSIONES}

A partir de los resultados obtenidos, de su análisis y discusión, se pueden extraer las siguientes conclusiones principales:

1) El sistema desarrollado es una propuesta tecnológica que busca disminuir la brecha existente al rechazo de planes de negocios realizados por emprendedores o microemprensarios que buscan financiamiento público 0 privado, con la finalidad de potenciar los procesos de su empresa.

2) Los servicios que se ofrecen y en particular el servicio de coaching que apoyarán en línea a los emprendedores, validándoles cada documento que haya decidido trabajar. El emprendedor será orientado a través de tips o mensajes de ayuda que se deben considerar para la generación de un correcto plan de negocio y, de esta forma, mejorar la probabilidad de obtener un financiamiento público o privado.

3) Estos servicios, en las pruebas de aceptación, demostraron ser eficaces ya que la misma empresa Kuvemar, es un emprendimiento tecnológico y que presta asesoría a emprendedores de la Región de Arica-Parinacora en Chile.

4) La herramienta desarrollada pueda generar nuevas oportunidades de negocios, especialmente para emprendedores desorientados en la materia, y no solo en el territorio nacional, sino también en el internacional, dado a que las funcionalidades de la herramienta permiten estructurar y generar cualquier tipo de documento que haga referencia a algún fondo concursable. 
5) El sistema tiene gran potencial para ser utilizado por alumnos y profesores de la Universidad de Tarapacá, debido a que distintas carreras, como Ingeniería en Computación e Informática, Ingeniería Mecánica, Ingeniería Industrial, entre otras, tienen asignaturas de emprendimiento en donde realizan modelos de negocios, como también documentos que representan la matriz FODA (fortalezas, oportunidades, debilidades, amenazas) de una idea de negocio. Se cuenta con el apoyo desinteresado de la empresa Kuvemar, para dar soporte.

6) El sistema fue desarrollado aplicando tecnologías modernas y una metodología evolutiva incremental, representada mediante casos de uso que incluye las etapas de especificación de requisitos, análisis y diseño, implementación y pruebas del software a través del estándar IEEE 1012-2016.

\section{AGRADECIMIENTOS}

Este trabajo cuenta con el financiamiento del programa de Investigación de la Universidad de Tarapacá, Arica, Chile (2017-2018), en el contexto de los proyectos código 8742-17 y código 8727-18. Asimismo, a la empresa Kuvemar por su valiosa colaboración, interés y disposición de los equipos computacionales para el desarrollo, prueba y puesta en servicio del sistema construido.

\section{REFERENCIAS}

Alarcón-Aldana, A., Urrutia-Pinilla, J., \& Callejas-Cuervo, M., Aplicación Móvil para la Administración de Variables Físicas en Ciclismo al Aire Libre, Información tecnológica, 27(4), 175-182 (2016).

Amorós, J. E., Abarca, A. y Mandakovic, V., Global Entrepreneurship Monitor Reporte Nacional de Chile 2015, GEM, Santiago, Chile (2015).

Cabarcas, A., Puello, P., y Martelo, R., Sistema de Información Soportado en Recuperación XML para Pequeñas y Medianas Empresas (PYME) de Cartagena de Indias, Colombia, Información tecnológica, 26(2), 135-144 (2015).

Castro-Rivera, V., Herrera-Acuña R., y Villalobos-Abarca, M., Desarrollo de un Software Web para la generación de planes de gestión de riesgos de software. Información tecnológica, 31(3), en prensa (2020).

Cifuentes, O., Aldana, F, y Aya, A. Y., UNbizplanner: herramienta de software para preparar planes de negocios, Ingeniería e Investigación, 28(1), 154-161 (2008).

Coca, G. A., Castrillón, O. D., y Ruiz-Herrera, S., Programación de un Sistema de Fabricación tipo "Job Shop" bajo un Enfoque de Sostenibilidad, Información tecnológica, 27(6), 31-52 (2016).

CORFO, Resultados Primera Etapa Capital Semilla Primera Convocatoria 2018, Ministerio de Economía, Fomento y Turismo, Santiago, Chile (2018).

E.S. Plan, E.S. Plan de Empresa [En línea]. Available: http://www.planempresa.pro/. [Último acceso: 10 de Enero de 2020].

Ferreira-Herrera, D. C., El modelo Canvas en la formulación de proyectos, Cooperativismo \& Desarrollo, 23(107), (2015).

Fino-Garzón, D. M., Innovación en modelos de negocio: metodología Lean Canvas en una startup de base tecnológica, E-Lis Repaository (2013).

Gómez, U. E., Pérez, J. P., y Ramírez, J. L., Sistema de Información Agrícola para la disminución de Brechas entre Oferta y Demanda-AGROCRAFT, Información tecnológica, 27(3), 215-220 (2016).

Herrera-Acuña, R., y Villalobos-Abarca, M. Caracterización de la Formación Académica en Emprendimiento de Pregrado que se realiza en Universidades Chilenas, Formación universitaria, 11(4), 43-52 (2018).

Kim, J. K., Woo, J. B., Kim, S. J., Kim, H. W., Ro, Y. S., Choi, J. J., y Park, J. H. Application Study for Implementing IEEE 1012-2016. Transactions of the Korean Nuclear Society Spring Meeting, Jeju, Korea (2017)

Krell, R., Boletín: El Microemprendimiento en Chile, Ministerio de Economía, Fomento y Turismo, Santiago, Chile (2018).

Kuvemar, Kuvemar Desarrollo Tecnológico, https://www.kuvemar.com (2020).

Mamani, M., Villalobos, M., \& Herrera, R., Sistema web de bajo costo para monitorear y controlar un invernadero agrícola, Ingeniare, 25(4), 599-618 (2017).

Maurya, A., Scaling lean: Mastering the key metrics for startup growth, Penguin Books, USA (2016).

Ocampo, M. A. T., Nova, L. D., y Sanabria, I. V. B., importancia de las pymes para el desarrollo económico de chile en el siglo XXI, Punto de Vista, 9(14) (2018).

Osterwalder, A., y Pigneur, Y., Business model generation: a handbook for visionaries, game changers, and challengers, John Wiley \& Sons, Hoboken, New Jersey, USA (2010).

Quintero, W., Robles, C. A., y Viloria, A. M., Sistema de Información para Detección de Crecientes Súbitas en la Cuenca del Río Manzanares en Santa Marta, Colombia, Información tecnológica, 28(6), 95-102 (2017). 
Thomsen, M. y Gerard, F., El Plan de Negocios Dinámico: Como iniciar un negocio. Thomsen Business Information, España (2009).

Toro-Jarrín, M. A., Ponce-Jaramillo, I. E., y Güemes-Castorena, D., Methodology of the building process integration of Business Model Canvas and Technological Roadmap, Technological Forecasting and Social Change, 110, 213-225 (2016).

Trías de Bes, F., El libro negro del emprendedor: no digas que nunca te lo advirtieron, Empresa Activa, Ediciones Urano, Argentina (2007).

Vidal, C., López, L., Rojas, J., y Castro, M., Desarrollo de Sistema Web de Reclutamiento y Selección y de Directivos por Competencias mediante PHP Codelgniter 3.0. Información tecnológica, 28(2), 203-212 (2017). 
